\title{
The Mechanism of Reinforced Backfill Body with Flexible Mesh
}

\author{
Xiaosheng Liu $\mathbb{D}^{1,2}$ Weijun Wang, ${ }^{1}$ Quan Liu, ${ }^{2}$ and Chao Yuan ${ }^{1}$ \\ ${ }^{1}$ School of Resources, Environment and Safety Engineering, Hunan University of Science and Technology, Xiangtan 411201, China \\ ${ }^{2}$ Changsha Institute of Mining Research Co., Ltd., Changsha 410012, China
}

Correspondence should be addressed to Xiaosheng Liu; 13681996@qq.com

Received 11 January 2021; Revised 24 February 2021; Accepted 16 March 2021; Published 2 April 2021

Academic Editor: Qi Jia

Copyright (C) 2021 Xiaosheng Liu et al. This is an open access article distributed under the Creative Commons Attribution License, which permits unrestricted use, distribution, and reproduction in any medium, provided the original work is properly cited.

The backfill of metal mines is easily damaged by the disturbance due to their low strength. We proposed a method that uses flexible meshes as the backfill skeleton to enhance the strength of the backfill. The physical and mechanical properties of the flexible meshreinforced filling body are investigated by combining theoretical analysis and laboratory experiments. The strengthening effect is remarkable with the flexible meshes. With the friction-passive resistance between the high-strength reinforcement material and the filling body, the insufficient tensile strength of the filling body is compensated and the reinforcement is improved. The ultimate compressive strength is increased by 1.07 to 1.35 times, and the elastic modulus is increased by 1.08 to 4.42 times. We concluded that the essence of strengthening the flexible mesh-reinforced filling is to increase the cohesive force of the filling and increase the ability to resist external load damage.

\section{Introduction}

Mine backfill is the cementing of tailings, cement, and other aggregates into the excavation area. Backfilling is an indispensable part of mine operation to stabilize mine stress concentration and dispose of mine waste. Generally, there are three types of backfill, including the hydraulic fill (slurry and dense slurry fills), the paste fill (coarse tailings, medium tailings, and fine tailings), and the rock fill (cemented and noncemented) [1]. These three types are adapted to different mining scenarios. How to improve the strength of the filling body and whether it is meaningful to reinforce the filling body with filling body mesh are still unclear. This motivated our study on the mechanical characteristics of reinforced backfill body with flexible mesh.

Annor (2002) studied the characteristics and behavior of composite backfill material. Yin et al (2019) provided an innovative method for the placement of gangue backfilling material in steep underground coal mines [2]. They found when the strength of the backfill body does not meet the requirements, flexible materials or steel mesh will be added according to the site conditions.

Reinforced strengthening technology refers to the technology that mixes or implants reinforced materials with relatively high-strength and elastic modulus into the reinforced body to improve the mechanical properties of the reinforced body, represented by the reinforced soil technology. After a long time of development of reinforced soil technology, the theory and application have achieved fruitful results. For example, Wang Zhijie [3], Zhou Bin et al. [4], and He Zhijun et al. [5] have proved that geogrid can significantly improve the maximum axial stress of reinforced soil through large-scale biaxial compression test; Shi Youzhi [6] concluded that the reinforced soil interface is a weak sliding surface through the pull-out test of different reinforced materials and considered that the interface antisliding stability should be calculated in the reinforced soil engineering design; and Lei Shengyou et al. [7] carried out damage soil. The results show that the strength of reinforced soil is the highest, that of plain soil is the second, and that of damaged soil is the least. In practical applications, the use of geogrids (Wang et al., 2019), geotextiles (Chang L., 2019), gravel (Liu W., 2019), and fibers (Hu Y., 2019) to reinforce roadbeds, dams, slopes, and retaining walls has become a common method and approach [8-11].

However, due to the late start of backfill reinforcement technology, there are relatively few studies and applications of reinforcement technology theory in the field of mine 
filling. For example, Bernie (2017) studied engineering backfill fiber as an environmental solution to cost reduction [12]. Liu X. (2019) investigated the filling method of the suspended reinforced filling body [13]. Liu Q. (2019) studied the mechanical properties and reinforcement of the filling body with weak interlayer [14].

But the research on the reinforcement of filling bodies is mostly fiber reinforcement. Deng (2005) studied the mechanical properties, damage, and stability of the filling body in extralarge stope of Anqing copper mine [15]. Sun et al. (2018) performed an experimental study on the mechanical properties of polypropylene fiber high water materials [16]. Ma et al. (2016) and Li et al. (2016) carried out the macroand microtests of fiber-reinforced paste filling materials $[17,18]$. Yi et al. (2015) and Yi et al. (2018) studied the compressive behavior of fiber-reinforced cemented paste backfill $[19,20]$.

Although a relatively optimistic effect has been achieved in enhancing the strength of the filling body, fiber reinforcement increases the strength of the filling body while also reducing the fluidity of the filling slurry, which brings new challenges to the efficient activation preparation and long-distance transportation of filler slurry.

In view of the problems mentioned above, this paper proposes a new reinforcement technology that can solve the difficulties in preparing filler slurry and long-distance transportation caused by fiber reinforcement. It is a composite body with flexible mesh as the tensile and flexible framework of the backfill. The higher tensile performance of the three-dimensional flexible grid and the compressive performance of the filling body complement each other, which improves the overall strength and stability of the filling body. This paper uses a combination of theory and laboratory experiments to analyze the strength and degeneration characteristics of the flexible grid-reinforced filling body and study its strengthening mechanism.

\section{Experiment}

2.1. Materials and Equipment. The reinforced material flexible grid is made of polyethylene. The specifications are as follows: the diameter of the rope is $1 \mathrm{~mm}$, the tensile strength of the single rope is $15 \mathrm{~kg}$, the mesh size is $20 \mathrm{~mm} \times 20 \mathrm{~mm}$, and the layer spacing is $20 \mathrm{~mm}$.

The filler slurry aggregate adopts the whole tailings of a gold mine, the specific gravity is 2.71 , the loose bulk density is $1.07 \mathrm{t} / \mathrm{m}^{3}$, the average particle size is $63 \mu \mathrm{m}$, and the 200 mesh accounts for $70 \%$. The tailings are medium and coarse tailings; the filling cementing material is Hunan Changsha Pingtang Cement Factory produces P.O42.5 ordinary Portland cement.

The test loading equipment uses a 60-ton YNS-Y600 electrohydraulic servo rigid control material testing machine to perform uniaxial compression tests on the specimens with a test accuracy of $0.001 \mathrm{kN}$. The equipment has multiple loading control methods of force, deformation, displacement, stress, and strain and can realize impact-free conversion among various methods during the test. The load sensor is used to directly measure the force, which can overcome the disadvantages of the influence of the piston friction of the hydraulic sensor, and the measurement is accurate. The maximum loading capacity is $600 \mathrm{KN}$. The displacement measurement error is less than $0.5 \%$.

2.2. Test Program. In order to reduce the influence of the size effect on the test results, a cube test block with a size of $100 \mathrm{~mm} \times 100 \mathrm{~mm} \times 100 \mathrm{~mm}$ was made. A total of 6 sets of tests were prepared, with 3 test blocks in each group, numbered S1 S3 (without flexible grid-reinforced) and J1 $\sim$ J3 (flexible grid-reinforced). The parameters are designed as shown in Table 1.

According to the test design, a total of 18 filling body test blocks are made, and the manufacturing process of reinforced filling body test block is shown in Figure 1. The molding and demoulding test block was moved to a constant temperature and humidity curing box with a temperature of $(20 \pm 2)^{\circ} \mathrm{C}$ and humidity of $(95 \pm 5) \%$ and cured for 28 days, as shown in Figure 2.

The loading method of test is displacement loading, and the loading rate is controlled at $0.5 \mathrm{~mm} / \mathrm{min}$. The loading process of the specimen is shown in Figures 3 and 4.

\section{Results}

3.1. Mechanical Properties of the Filling Body. The stressstrain curve of the filled test block is shown in Figure 4. The ultimate compressive strength and elastic modulus of the filling body after reinforcement have been improved to a certain extent, especially for the strength of the filling body in the postdestructive stage, as shown in Table 2.

The ultimate compressive strength and elastic modulus of the J1 group increased by $0.14 \mathrm{MPa}$ and $0.027 \mathrm{GPa}$, respectively. The strengthening coefficients were 1.07 and 1.08, respectively. The ultimate compressive strength and elastic modulus of the J2 group increased by $0.098 \mathrm{MPa}$ and $0.025 \mathrm{GPa}$, respectively. The strengthening coefficients were 1.20 and 1.41. The ultimate compressive strength and elastic modulus of the J3 group increased by $0.104 \mathrm{MPa}$ and $0.032 \mathrm{GPa}$, respectively, and the strengthening coefficients were 1.36 and 1.81 , respectively.

This improvement of elastic modulus is obvious when the lime-sand ratio is relatively small (i.e., when its own compressive strength is low), and when the lime-sand ratio is relatively large (the compressive strength is higher), the improvement is limited. Of course, the precision of the improvement may relate to the calculation method. But since we used the same method when calculating the two experiments (with and without mesh), the relative improvement is reliable.

3.2. Failure Mode. The failure modes of the backfill samples before and after reinforcement are quite different. The failure mode of the backfill before reinforcement is affected by various factors and the mode is complex, mainly including large shear failure, tensile failure, tension-shear composite failure, and slip failure. The failure mode of the filling body 
Table 1: Parameter design of reinforced backfill.

\begin{tabular}{lccccc}
\hline Number & Cement sand ratio & Concentration (\%) & Number & Cement sand ratio & Concentration (\%) \\
\hline S1 & $1: 4$ & 66 & J1 & $1: 4$ & 66 \\
S2 & $1: 10$ & 66 & J2 & $1: 6$ & 66 \\
S3 & $1: 15$ & 66 & J3 & $1: 8$ & 66 \\
\hline
\end{tabular}

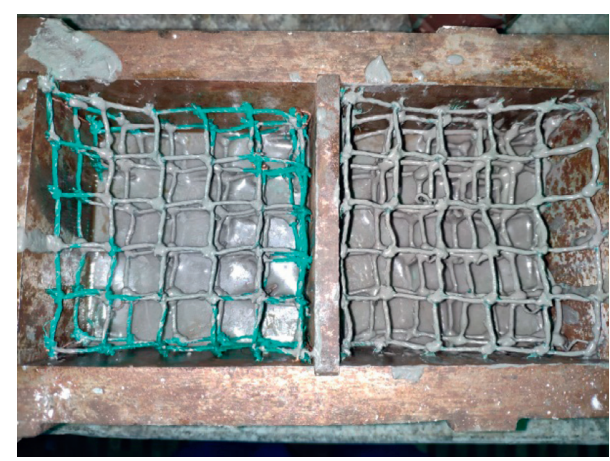

FiguRE 1: 3D flexible grid reinforcement of filling body.

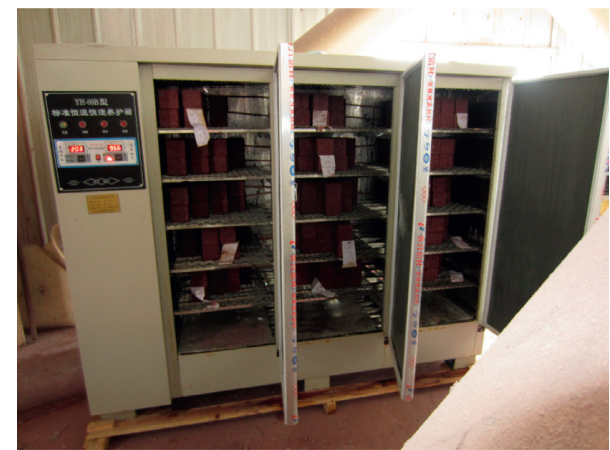

Figure 2: Curing of constant temperature and humidity test block for filling body.

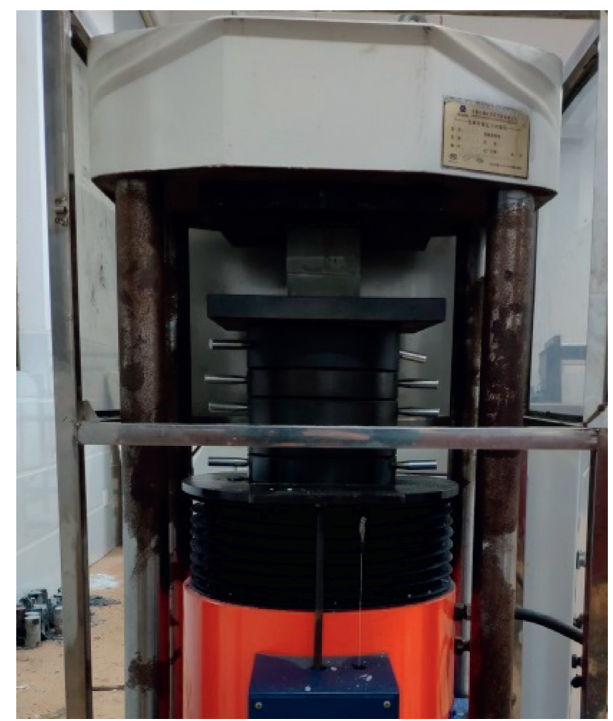

FIGURE 3: Loading process of backfill specimen. after reinforcement is relatively simple and mainly dominated by small shear failure, as shown in Figure 5 .

The flexible three-dimensional mesh layer is closely connected, and the force transmission characteristics are better. At the same time, the shear strength of the mesh ribs is higher than the shear failure strength of the filling body, and the shear stress cannot cut the flexible ribs to form a large shear surface. Under the action of the flexible threedimensional grid, the internal stress distribution of the filling body changes, the stress concentration is reduced, and largearea shear failure is avoided, which indirectly improves the load-bearing strength of the filling body. Therefore, the flexible three-dimensional grid-reinforced filling body is subjected to axial compression, from local large-area failure to relatively uniform small shear failure, and the outline of the filling body remains relatively complete after the failure and the surrounding fragments caused by shear failure. The block size is relatively uniform, and there will be no peeling of large blocks.

3.3. Deformation Characteristics. The deformation characteristics of the reinforced backfill are generally similar to those of the plain backfill. The difference is only reflected in the postpeak failure stage. The reinforced backfill exhibits more obvious hardening in the postpeak failure stage. The deformation characteristics can be summarized into five stages: (1) in the compaction stage, the filling body undergoes void compaction and structural surface closure; (2) in the elastic deformation stage, the stress and strain at this stage are linearly related, and this stage is affected by the interlayer and changes; (3) in the plastic deformation stage, the filling body occurs in this stage plastic deformation and cracks begin to occur; (4) in the strain softening stage, the stress at this stage decreases with the increase of the strain of the filling body; and (5) in the strain hardening stage, the stress at this stage increases with the increase of the compression deformation of the filling body specimen, and there is a tendency of exceeding the ultimate failure strength.

\section{Discussion}

4.1. Mechanical Strengthening Mechanism. The strengthening mechanism of the flexible grid is the coupling effect between the grid and the filling. The tensile and noncompressive properties of the flexible grid make up for the low tensile strength of the reinforced body and improve the resistance of the filling to damage, as shown in Figure 6. The force of the longitudinal ribs and the interlayer connecting ribs of the reinforced filling body is transmitted to the adjacent longitudinal ribs and the vertical transverse ribs through the connecting nodes between the ribs. 


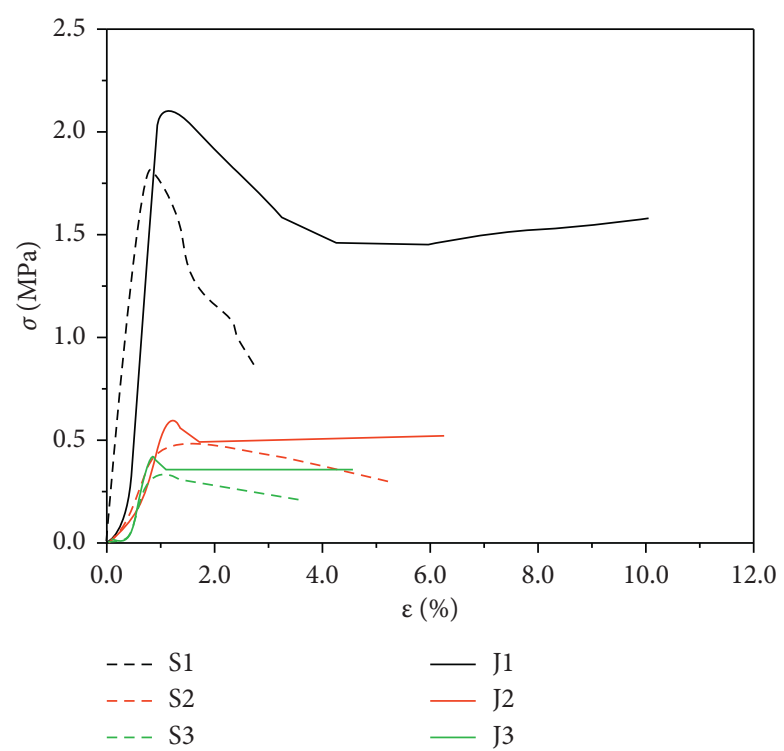

FIGURE 4: Stress-strain curve of backfill before and after reinforcement.

TABLE 2: Comparison of some mechanical parameters of backfill before and after reinforcement.

\begin{tabular}{lcccc}
\hline \multirow{2}{*}{ Ratio } & \multicolumn{2}{c}{ Without flexible grid-reinforced } & \multicolumn{2}{c}{ Flexible grid-reinforced } \\
& Ultimate strength $(\mathrm{MPa})$ & Elastic modulus $(\mathrm{GPa})$ & Ultimate strength $(\mathrm{MPa})$ & Elastic modulus $(\mathrm{GPa})$ \\
\hline $1: 4$ & 1.88 & 0.29 & 2.02 & 0.31 \\
$1: 10$ & 0.49 & 0.06 & 0.59 & 0.08 \\
$1: 15$ & 0.29 & 0.04 & 0.39 & 0.07 \\
\hline
\end{tabular}
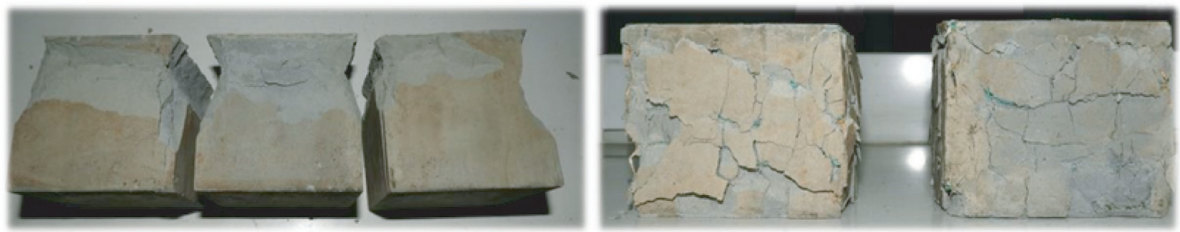

FIGURE 5: Comparison of filling failure modes before and after reinforcement.

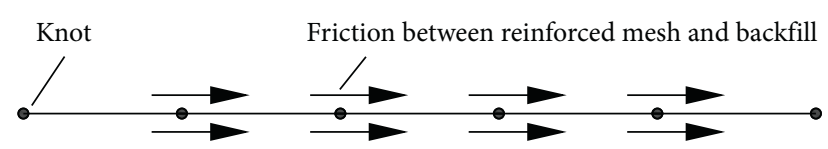

End bearing capacity of filling body and transverse reinforcement of reinforced mesh

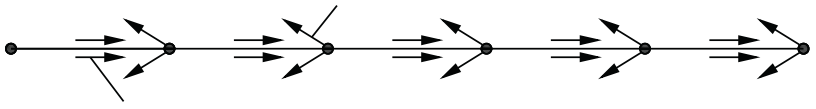

Friction force between filling particles inside and outside reinforced mesh

FIgURE 6: Coupling relationship between flexible grid and stiffener.

The ribs may be pulled out to cause displacement under tension. However, the ribs are squeezed under the action of the filling body's own weight and external load, and the friction and biting force between the filling body and the grid prevent the grid and the filling body from generating relative displacement. The force model of the flexible grid is shown in Figure 7.

According to the theoretical model of stiffened force, the force and deformation of the stiffened body lead to the force of each force element:

$$
\Delta T=T_{z}-T_{y},
$$

where $T_{z}$ and $T_{y}$ are, respectively, the lateral deformation and tension transferred from the connection knots of the two ends of the force element to the brace.

Under the action of external load and dead weight, the total frictional resistance between the filling body of the force-bearing element and the tendons is

$$
F=\pi \sigma_{y} d L \mu,
$$

where $L$ is the length of the force receiving unit, $\mu$ is the friction factor of the contact surface between the brace and the filling body, and $d$ is the diameter of the rib. 


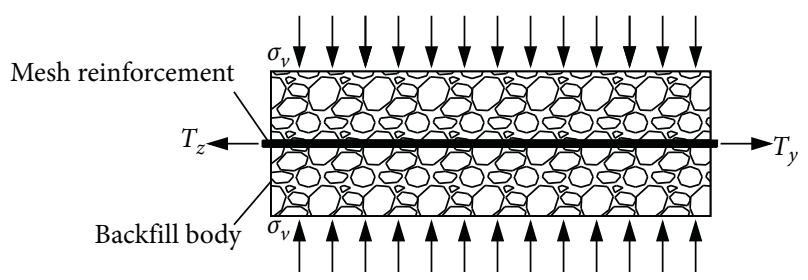

FIgURE 7: Stress model of flexible grid reinforcement.

According to the principle of mechanical balance, when $F$ is less than $\Delta T$, there will be a relative displacement between the rib and the filling body; when $F$ is greater than $\Delta T$, the relative balance between the rib and the filling body will not be displaced, and the force-bearing unit body remains stable. Therefore, the flexible grid-reinforced filling body should meet the following necessary conditions: (1) the surface of the reinforced material is rough enough to generate greater antisliding friction between the reinforcement mesh and the filling body; (2) the reinforced material has higher tensile strength and the elastic modulus constitute the conditions for the relative displacement trend; and (3) the filling body has sufficient density to produce sufficient interaction force with the reinforced material.

\subsection{Mechanical Strength Characteristics of Reinforced Backfill.} Reinforced backfill is a material composite with different material properties, and the elastic modulus of the reinforced material is much greater than that of the filling body. Therefore, the coupling effect of the reinforcement material and the filling body includes the shear resistance of the filling body, the synergistic effect of the friction resistance of the reinforcement, and the tensile strength of the reinforcement.

Under the Poisson effect, the lateral deformation of the reinforced backfill exerts a lateral pulling force on the reinforcement. At the same time, the lacing bars will exert a force in the opposite direction on the filling body to limit the lateral expansion and deformation of the filling body. The lateral restraint force reduces the lateral deformation pressure of the stiffened backfill. Under the same axial deformation, the minimum principal stress borne is smaller than that of the plain backfill, as shown in Figure 8. In Figure 8, (a) is the molar circle in the undamaged state of the plain backfill and (b) is the molar circle in the ultimate failure state of the plain backfill.

According to the Mohr-Coulomb theory, the relationship between $\sigma_{1 f}$ and $\sigma_{3 f}$ in the limit equilibrium state is

$$
\sigma_{1 f}=2 c \tan \left(\frac{\varphi}{2}+\frac{\pi}{4}\right)+\sigma_{3 f} \tan ^{2}\left(\frac{\varphi}{2}+\frac{\pi}{4}\right) .
$$

However, for reinforced fillings, under external load conditions, in addition to transverse and longitudinal stresses, the fillings are also subjected to the lateral force $T$ of the reinforced material and the reaction force $R$ of the fillings. The subisolator with broken body structure is subjected to force analysis, and the force is shown in Figure 9.

The reinforcement of the backfill adopts a flexible mesh. The interaction effect of the horizontal, vertical, and

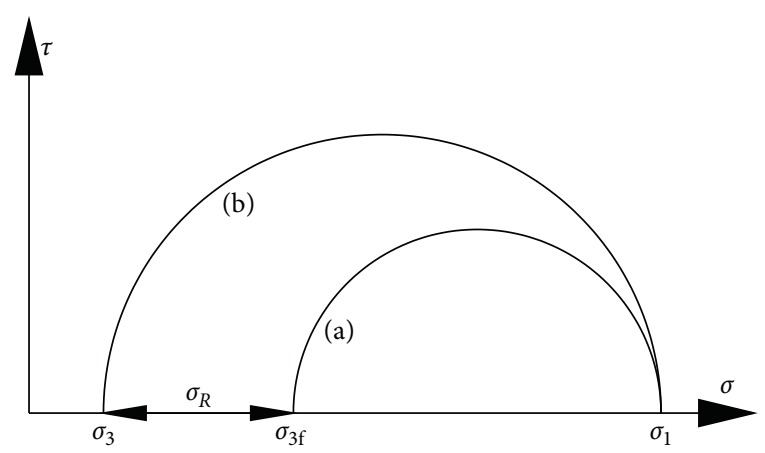

FIGURE 8: Variation of lateral stress of filling body under the same normal stress.

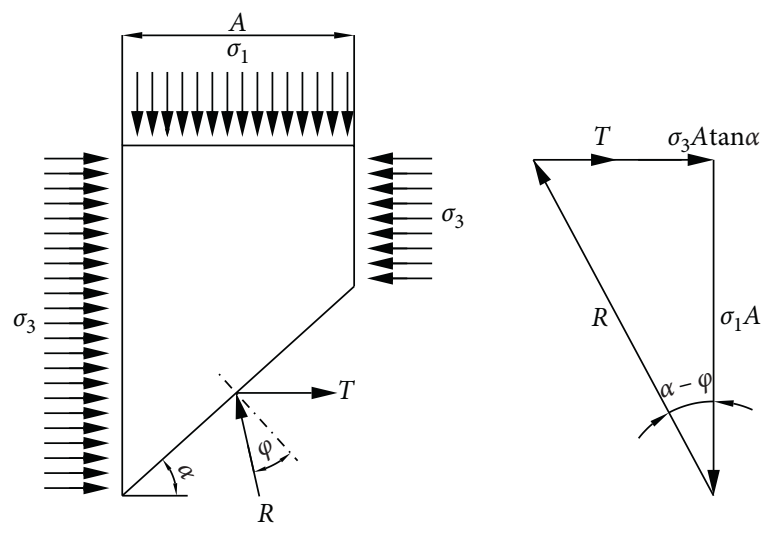

FIgURE 9: Mechanical balance diagram of reinforced backfill.

interlayer connecting ribs of the flexible mesh is good. Reinforced backfill is only subjected to the limit state analysis of tensile strength. Assuming that the angle between the fracture surface of the reinforced filling body and the horizontal plane is $\alpha$, according to the static balance principle, we can obtain

$$
T+\sigma_{3} A \tan a=R \sin (\alpha-\varphi),
$$

where the tensile force of the brace can be calculated by

$$
T=\frac{R_{t} \cdot A \cdot \tan \alpha}{\Delta L \cdot \Delta H}
$$

where $\Delta H$ is the vertical layer spacing of the three-dimensional flexible grid; $\Delta L$ is the horizontal layer spacing of the three-dimensional flexible grid; and $R_{t}$ is the tensile strength of the flexible ribs.

In formula (4), the reaction force $R$ of the backfill can be calculated by the following formula:

$$
R=\frac{\sigma_{1} \cdot A}{\cos (\alpha-\varphi)}
$$

Substituting formula (6) and formula (5) into formula (4), we obtain

$$
\sigma_{1}=\left(\sigma_{3}+\frac{R_{t}}{\Delta L \cdot \Delta H}\right) \tan \alpha \times \cot (\alpha-\varphi) .
$$




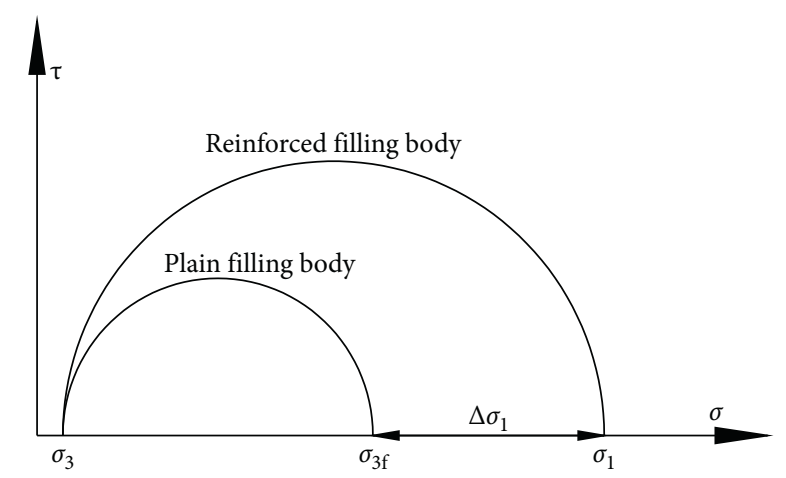

FIGURE 10: Variation of normal stress of backfill under the same side stress condition.

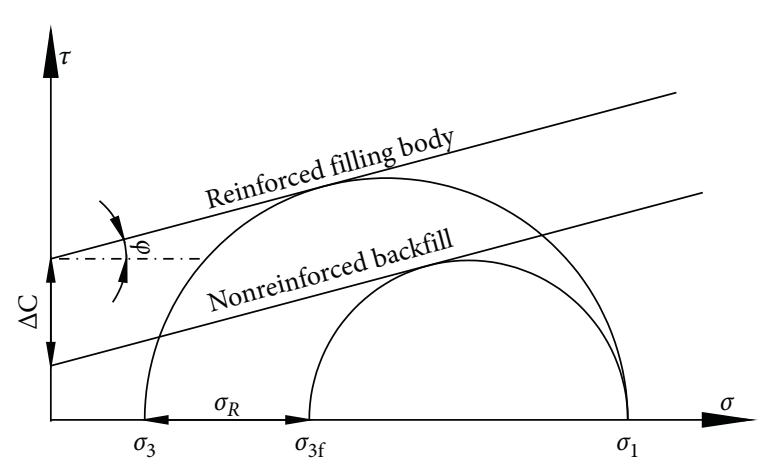

FIGURE 11: Under the same normal stress condition, the shear strength and lateral stress changes of backfill.

When $\sigma_{1}$ is the maximum principal stress, then $\alpha=45^{\circ}+(\varphi / 2)$, and

$$
\sigma_{1}=\sigma_{3} \tan ^{2}\left(45^{\circ}+\frac{\varphi}{2}\right)+\frac{R_{t}}{\Delta L \cdot \Delta H} \tan ^{2}\left(45^{\circ}+\frac{\varphi}{2}\right) .
$$

Comparing formula (3) and formula (8), it can be found that, under the limit equilibrium condition of the reinforced backfill structure, the linear relationship between the first principal stress and the third principal stress is maintained. Reinforcing does not affect the friction angle of the filling body. Under the condition of the minimum principal stress $\sigma_{3}$, the maximum principal stress $\sigma_{1}$ that can be reached when the backfill fails is increased by a fixed value $\sigma_{R}$ compared with the plain backfill, which is the initial stress of the reinforced backfill, as shown in the following equation:

$$
\sigma_{R}=\frac{R_{t}}{\Delta L \cdot \Delta H} \tan ^{2}\left(45^{\circ}+\frac{\varphi}{2}\right)-2 c \cdot \tan \left(45^{\circ}+\frac{\varphi}{2}\right) .
$$

The relationship between the shear stress and the normal stress on the fracture surface can be expressed by the following formula:

$$
\tau=\sigma_{1} \tan \varphi+\frac{R_{t}}{2 \Delta L \cdot \Delta H} \tan \left(45^{\circ}+\frac{\varphi}{2}\right) .
$$

It can be seen from equation (10) that the shear strength of the reinforced backing body is increased by an increment equal to $\Delta C$ compared with the shear strength of the unreinforced backing body. This is the cohesive force generated by the reinforced material; that is, the tensile strength generated by the reinforced material corresponds to the molar stress circle of the reinforced and unreinforced filling body, which is the increment of the shear strength line intercept $\Delta C$, as shown in the following equation:

$$
\Delta C=\frac{R_{t}}{2 \Delta L \cdot \Delta H} \tan \left(45^{\circ}+\frac{\varphi}{2}\right)-c .
$$

When the unreinforced plain backfill reaches the limit equilibrium under the action of $\sigma_{3 f}$ and $\sigma_{3 f}$, the reinforced backfill will not be damaged. After the filling body is reinforced, the maximum principal stress increases from $\sigma_{3 f}$ to $\sigma_{1}$ to reach the ultimate equilibrium state, the maximum principal stress increases, and the resistance of the filling body to damage is significantly enhanced, as shown in Figure 10. Based on the principle of constant internal friction angle and increased cohesive force after the filling body is reinforced, the maximum principal stress remains unchanged. After the filling body is reinforced, the minimum principal stress will decrease $\sigma_{R}$, which strengthens the damage ability of the backfill against lateral pressure, as shown in Figure 11.

\section{Conclusions}

(1) The reinforcement mechanism of the flexible threedimensional mesh reinforcement of the filling body is the coupling effect of the reinforcement and the filling body. Through the friction-passive resistance mechanism between the high-strength reinforcement material and the filling body, the insufficient tensile strength of the filling body is compensated and the reinforcement is improved. In terms of strength characteristics, the reinforced filling body improves the cohesion of the backfill without changing the friction angle of the original backfill, so that the backfill obtains higher resistance to external load strength.

(2) The three-dimensional flexible mesh reinforcement of the backfill effectively improves the mechanical properties of the filling body, and the ultimate compressive strength and elastic modulus are improved to a certain extent. At the same time, the backfill has the ability of strain hardening in the later stage of failure. The increased rupture of the filling body maintains greater residual strength, and there is a tendency to continue to grow beyond the ultimate compressive strength.

(3) The reinforcement of the flexible mesh of the backfill changed the failure mode of the filling body, improved the internal stress distribution when the filling body is stressed, eliminated the local stress concentration, avoided the large shear failure of the filling body, and played the role of indirect strengthening of the backfill [21]. 


\section{Data Availability}

Data used to support the findings of this study are available on request to the corresponding author.

\section{Conflicts of Interest}

The authors declare that they have no conflicts of interest.

\section{References}

[1] D. O'Toole, "The basics of mine backfill," Engineering and Mining Journal, vol. 205, no. 12, p. 27, 2004.

[2] Y. Yin, T. Zhao, Y. Zhang et al., "An innovative method for placement of gangue backfilling material in steep underground coal mines," Minerals, vol. 9, no. 2, p. 107, 2019.

[3] Z. Wang, F. Jacobs, and M. Ziegler, "Influence of geogrid transverse rib on strength and deformation characteristics of reinforced gravel," Geotechnical Mechanics, vol. 38, no. 8, pp. 2234-2240, 2017.

[4] B. Zhou, T. Ling, Z. Zheng, and B. Yang, "Large scale triaxial test of geogrid reinforced soil composite," Journal of Chongqing Jiaotong University, vol. 31, no. 4, pp. 792-794, 2012.

[5] Z. He and J. Zou, "Large scale triaxial test analysis of reinforced fine-grained soil sand," Journal of Railway Science and Engineering, vol. 16, no. 10, pp. 2451-2458, 2019.

[6] Y. Shi, "Engineering characteristics of geogrid and application of reinforced cushion," Dissertation, Huaqiao University, Quanzhou, China, 2003.

[7] S. Lei, "Study on strength of reinforced soil considering reinforcement damage," Journal of Three Gorges University, vol. 32, no. 6, pp. 42-46, 2010.

[8] N. Wang, W. Chen, and D. Zhu, "Analysis of reinforcement effect of geotechnical reinforcement on open pit dump slope," Coal Mine Safety, vol. 50, no. 5, pp. 281-284, 2019.

[9] L. Chang, "Construction method and engineering application of reinforced earth retaining wall for high subgrade," Sichuan Architecture, vol. 39, no. 2, pp. 315-317, 2019.

[10] W. Liu, "Application of reinforced earth retaining wall in an overseas project," Energy Saving in Nonferrous Metallurgy, vol. 35, no. 2, pp. 50-53, 2019.

[11] Y. Hu, "Application of reinforced earth retaining wall in flood control and disaster reduction revetment engineering construction," Shaanxi Water Conservancy, vol. 8, pp. 189-190, 2019.

[12] B. Ting, "Engineering backfill fiber as an environmental solution to cost-reduce and improve paste backfill," in Proceedings of the 2017 International Conference on Mining with Backfill, Denver, Co, USA, July 2017.

[13] X. Liu, W. Wang, W. Lai, L. Jia, C. Liu, and D. Mo, "Gob Filling method of suspended reinforced filling body," 2019.

[14] Q. Liu, "Study on mechanical properties and reinforcement of filling body with weak interlayer," Dissertation, Changsha Institute of Mining Research, Changsha, China, 2019.

[15] D. Deng, "Study on mechanical properties, damage and stability of filling body in extra large stope of anqing copper mine," 2005.

[16] W. Sun, C. Liu, W. fan, Z. Diao, F. Bo, and X. Li, "Experimental study on mechanical properties of polypropylene fiber high water materials," Metal Mine, vol. 1, pp. 53-57, 2018.

[17] G. Ma, Z. Li, X. Yi et al., "Macro and micro tests of fiber reinforced paste filling materials," Journal of Beijing University of Technology, vol. 42, no. 3, 2016.
[18] D. Li, Z. Xiong, Y. Wang et al., "Effects of air entraining agent and polypropylene fiber on properties of high water content materials," China Production Safety Science and Technology, vol. 12, no. 3, pp. 54-59, 2016.

[19] X. W. Yi, G. W. Ma, and A. Fourie, "Compressive behaviour of fibre-reinforced cemented paste backfill," Geotextiles \& Geomembranes, vol. 43, no. 3, pp. 207-215, 2015.

[20] X. W. Yi, G. W. Ma, and A. Fourie, "Centrifuge model studies on the stability of fibre-reinforced cemented paste backfill stopes," Geotextiles and Geomembranes, vol. 46, no. 4, pp. 396-401, 2018. 